Proceedings of the Edinburgh Mathematical Society (2007) 50, 37-47 (C)

DOI:10.1017/S0013091504000896 Printed in the United Kingdom

\title{
COMMUTATIVITY OF SKEW SYMMETRIC ELEMENTS IN GROUP RINGS
}

\author{
OSNEL BROCHE CRISTO AND CÉSAR POLCINO MILIES \\ Instituto de Matemática e Estatística, Universidade de São Paulo, Caixa Postal 66281, \\ 05315-970 São Paulo, Brazil (osnelier@ime.usp.br; polcino@ime.usp.br)
}

(Received 20 September 2004)

\begin{abstract}
Let $R$ be a commutative ring with unity and let $G$ be a group. The group $\operatorname{ring} R G$ has a natural involution that maps each element $g \in G$ to its inverse. We denote by $R G^{-}$the set of skew symmetric elements under this involution. We study necessary and sufficient conditions for $R G^{-}$to be commutative.
\end{abstract}

Keywords: group ring; skew symmetric elements; involution

2000 Mathematics subject classification: Primary 16U60

Secondary 16W10; 20C07

\section{Introduction}

Let $R G$ denote the group ring of a group $G$ over a commutative ring $R$. This ring has a natural involution * given by $\left(\sum \gamma_{g} g\right)^{*}=\sum \gamma_{g} g^{-1}$. Let

$$
R G^{-}=\left\{\gamma \in R G \mid \gamma^{*}=-\gamma\right\}
$$

be the set of skew symmetric elements of $R G$.

The problem of deciding when the symmetric elements of $R G$ commute or, equivalently, when they form a subring of $R G$ was studied in [1]. In this paper, we consider the problem of deciding when $R G^{-}$is commutative. The Lie product $[\gamma, \delta]=\gamma \delta-\delta \gamma$ induces a structure of Lie algebra in $R G^{-}$. The Lie nilpotency of this algebra was studied in $[\mathbf{2}, \mathbf{3}]$. The problem under consideration is to decide when the Lie product in $R G^{-}$is the trivial one, i.e. when $[\gamma, \delta]=0$, for all $\gamma, \delta \in R G^{-}$.

In what follows, we shall assume that $\operatorname{char}(R) \neq 2$ since if $\operatorname{char}(R)=2$, then $R G^{-}$coincides with the set of symmetric elements. In $\S 3$ we give a general answer to our question, except for the case $\operatorname{char}(R)=4$, which needs to be studied separately.

\section{Preliminary considerations}

Assume that $R$ is a commutative ring with unity and that $\operatorname{char}(R) \neq 2$. Note that if $g$ is any element in $G$, then $g-g^{-1} \in R G$ is skew symmetric. Actually, $R G^{-}$is generated, 
as an $R$-module, by the set

$$
\left\{g-g^{-1} \mid g \in G\right\}
$$

Thus, to study the commutativity of $R G^{-}$it will suffice to analyse the commutativity of elements of the form $g-g^{-1}$ among themselves.

Lemma 2.1. Let $g$ and $h$ be elements of the group $G$, such that $g^{2} \neq 1 \neq h^{2}$ and $\left[g-g^{-1}, h-h^{-1}\right]=0$. Then, one of the following holds:

(i) $g h=h g$;

(ii) $\left(g^{\alpha} h^{\beta}\right)^{2}=1$, for all $\alpha, \beta \in\{1,-1\}$;

(iii) $\operatorname{char}(R)=4$ and $\langle g, h\rangle \cong \mathcal{Q}_{8}$, the quaternion group of order 8 .

Proof. Suppose that $g h \neq h g$. Since

$$
0=\left[g-g^{-1}, h-h^{-1}\right]=g h-g h^{-1}-g^{-1} h+g^{-1} h^{-1}-h g+h g^{-1}+h^{-1} g-h^{-1} g^{-1},
$$

one of the following conditions holds:

(a) $g h=g h^{-1}$;

(b) $g h=g^{-1} h$;

(c) $g h=h^{-1} g^{-1}$;

(d) $g h$ is equal to at least two terms of the set $\left\{g^{-1} h^{-1}, h g^{-1}, h^{-1} g\right\}$, since char $(R) \neq 2$.

Note that cases (a) and (b) imply that $h^{2}=1$ and $g^{2}=1$, respectively, so they cannot occur. On the other hand, in case (c), $g^{-1} h^{-1}=h g$ holds and thus

$$
0=\left[g-g^{-1}, h-h^{-1}\right]=-g h^{-1}-g^{-1} h+h g^{-1}+h^{-1} g .
$$

So it follows that either $g h^{-1}=h g^{-1}$ or $g h^{-1}=h^{-1} g$. But $g h^{-1} \neq h^{-1} g$, because $g h \neq$ $h g$. Thus, $g h^{-1}=h g^{-1}$, and $g^{-1} h=h^{-1} g$. Hence, $\left(g^{\alpha} h^{\beta}\right)^{2}=1$, for all $\alpha, \beta \in\{ \pm 1\}$, and (ii) holds.

Finally, in case (d), we have $g h=g^{-1} h^{-1}=h g^{-1}=h^{-1} g$. In fact, if $g h=g^{-1} h^{-1}=$ $h g^{-1}$, then, as $g^{-1} h^{-1}=h g^{-1}$, it follows that $g h=h^{-1} g$. The other two possibilities in case (d) follow in a similar way. Hence, $\operatorname{char}(R)=4$ and

$$
\begin{aligned}
\langle g, h\rangle & =\left\langle g, h \mid g h=g^{-1} h^{-1}=h g^{-1}=h^{-1} g\right\rangle \\
& =\left\langle g, h \mid g^{4}=1, h^{2}=g^{2}, g h=h g^{-1}\right\rangle \\
& \cong Q_{8}
\end{aligned}
$$

and (iii) holds.

The lemma above gives some information that will be necessary to study the case when $\operatorname{char}(R) \neq 4$. For the special case when $\operatorname{char}(R)=4$, we shall need the following. 
Lemma 2.2. Assume that $R G^{-}$is commutative and let $g$ and $h$ be elements of $G$, such that $g^{2} \neq 1 \neq h^{2}$. Then, one of the following holds:

(i) $\langle g, h\rangle$ is abelian;

(ii) $\langle g, h\rangle=\left\langle g, h \mid g^{4}=h^{4}=(g h)^{2}=\left(g h^{-1}\right)^{2}=1\right\rangle$;

(iii) $\operatorname{char}(R)=4$ and $\langle g, h\rangle \cong\left\langle g, h \mid g^{8}=1, h^{2}=g^{4}, g h=h^{-1} g^{-1}\right\rangle$;

(iv) $\operatorname{char}(R)=4$ and $\langle g, h\rangle \cong \mathcal{Q}_{8}$.

Proof. We notice that, by Lemma 2.1, it is sufficient to assume that $\left(g^{\alpha} h^{\beta}\right)^{2}=1$, for all $\alpha, \beta \in\{1,-1\}$, and we have to show that (ii) and (iii) hold.

By applying Lemma 2.1 to the elements $g^{-1} h g$ and $h$, we see that $g^{-1} h g h=h g^{-1} h g$, or $\left(g^{-1} h g h\right)^{2}=1$, or $\operatorname{char}(R)=4$ and $\left\langle g^{-1} h g, h\right\rangle \cong \mathcal{Q}_{8}$.

Now we consider the case when $g^{-1} h g h=h g^{-1} h g$. As $h g=g^{-1} h^{-1}$, the left-hand side of the equation is equal to $g^{-2}$ and as $g^{-1} h=h^{-1} g$ the right-hand side is equal to $g^{2}$. Hence, we have $g^{-2}=g^{2}$, and $o(g)=4$. On the other hand, if $\left(g^{-1} h g h\right)^{2}=1$, in a similar way, we obtain $g^{-4}=1$ and again $o(g)=4$.

Finally, if $\operatorname{char}(R)=4$ and $\left\langle g^{-1} h g, h\right\rangle \cong \mathcal{Q}_{8}$, then $o(h)=4$ and $h^{-1}\left(g^{-1} h g\right) h=$ $\left(g^{-1} h g\right)^{-1}$. Thus, $h^{-1} g^{-2}=h g^{2}$ and $g^{4}=h^{-2}=h^{2}$.

Hence, either $o(g)=4$ or $\operatorname{char}(R)=4, o(h)=4$ and $g^{4}=h^{2}$. Similarly, by applying Lemma 2.1 to the elements $h^{-1} g h$ and $g$, we find that either $o(h)=4$ or $\operatorname{char}(R)=$ $4, o(g)=4$ and $h^{4}=g^{2}$. Thus, one of the following holds:

(a) $o(g)=4=o(h)$

(b) $\operatorname{char}(R)=4$ and either $o(g)=4$ and $h^{4}=g^{2}$, or $o(h)=4$ and $g^{4}=h^{2}$.

In the first case, (ii) holds. In the second case, (iii) holds, but one should note that we cannot claim equality in the statement of (iii), since the roles of $g$ and $h$ may have been interchanged. Thus, the lemma is proved.

\section{Skew symmetric elements when $\operatorname{char}(R) \neq 4$}

Notice that, when $\operatorname{char}(R) \neq 2,4$ or when $\operatorname{char}(R)=4$ and $G$ does not contain a copy of $\mathcal{Q}_{8}$, Lemma 2.1 leaves us only two options. We shall see that if condition (i) does not hold for some pair of elements $x, y \in G$ with $x^{2} \neq 1 \neq y^{2}$, then condition (ii) holds for all pairs of elements of $G$ with non-trivial squares.

Lemma 3.1. Assume that $R G^{-}$is commutative and that there exist elements $g, h \in G$, such that $g^{2} \neq 1 \neq h^{2}$ and $g h \neq h g$. If $\operatorname{char}(R) \neq 2,4$ or if $\operatorname{char}(R)=4$ and $G$ contains no subgroup isomorphic to $\mathcal{Q}_{8}$, then, for all $x, y \in G$ such that $x^{2} \neq 1 \neq y^{2}$, we have $\left(x^{\alpha} y^{\beta}\right)^{2}=1$, with $\alpha, \beta \in\{1,-1\}$.

Proof. Note that, by Lemma 2.1, we have $\left(g^{\alpha} h^{\beta}\right)^{2}=1$, for $\alpha, \beta \in\{1,-1\}$. Now, let $x \in G$ be such that $x^{2} \neq 1$; we will show that $(g x)^{2}=1$. 
Suppose that $(g x)^{2} \neq 1$. Thus, by Lemma 2.1, applied to $x$ and $g$, we have $x g=g x$. On the other hand, also by Lemma 2.1 , we have that either $x h=h x$ or $(x h)^{2}=1$. Again applying Lemma 2.1, to the elements $g x$ and $h$, we have two possibilities: either $g x h=h g x$ or $(g x h)^{2}=1$.

Assume that the first case holds. If $x h=h x$, then $g h x=g x h=h g x$, and thus $g h=h g$. On the other hand, if $(x h)^{2}=1$ then, as $\left(g h^{-1}\right)^{2}=1$, we have that $h g^{-1}=g h^{-1}$ and thus that $h g^{-1} x^{-1}=g h^{-1} x^{-1}=g x h=h g x$, where $g^{-1} x^{-1}=g x$. So, as $g^{-1} x^{-1}=x^{-1} g^{-1}$, it follows that $(g x)^{2}=1$. Hence, if $h g x=g x h$, then either $g h=h g$ or $(g x)^{2}=1$ : a contradiction.

Now, suppose that the other case holds; i.e. that $g x h=h^{-1} x^{-1} g^{-1}$. Then, as $x^{-1} g^{-1}=$ $g^{-1} x^{-1}$ and $g h=h^{-1} g^{-1}$, we have

$$
g x h=h^{-1} x^{-1} g^{-1}=h^{-1} g^{-1} x^{-1}=g h x^{-1},
$$

and $x h=h x^{-1}$. Thus, since we also have that either $x h=h x$ or $x h=h^{-1} x^{-1}$, it follows that either $h x=x h=h x^{-1}$ or $h^{-1} x^{-1}=x h=h x^{-1}$. So, either $x^{2}=1$ or $h^{2}=1$ : another contradiction.

Hence, $(g x)^{2}=1$ and, equivalently, $\left(g^{-1} x^{-1}\right)^{2}=1$. Similarly, taking $x^{-1}$, instead of $x$, it follows that $\left(g x^{-1}\right)^{2}=1$. Hence, $\left(g^{\alpha} x^{\beta}\right)^{2}=1$, for all $\alpha, \beta \in\{ \pm 1\}$.

Now, let $y \in G$ be such that $y^{2} \neq 1$. As $\left(x^{\alpha} g^{\beta}\right)^{2}=1$, for all $\alpha, \beta \in\{ \pm 1\}$, in order to show that $(x y)^{2}=1$, it is sufficient to work as above applying Lemma 2.1 to the elements $x y$ and $g$. Similarly, taking $y^{-1}$, instead of $y$, it follows that $\left(x y^{-1}\right)^{2}=1$.

Lemma 3.2. Let $R$ be a commutative ring with unity with $\operatorname{char}(R) \neq 2$ and let $G$ be any group. If one of the following conditions holds, then $R G^{-}$is commutative:

(i) $G$ is abelian;

(ii) $A=\langle g \in G \mid o(g) \neq 2\rangle$ is a normal abelian subgroup of $G$;

(iii) $G$ contains an elementary abelian 2-subgroup of index 2 .

Proof. If (i) holds, the statement is trivially true. On the other hand, if (ii) holds then $R G^{-}=R A^{-}$, since $(G \backslash A)^{2}=1$. So, as $A$ is abelian, it follows that $R G^{-}$is commutative.

Suppose now that (iii) holds, i.e. there exists a normal elementary abelian 2-subgroup $B$ of index 2 in $G$. In order to show that $R G^{-}$is commutative, it is sufficient to prove that $\left[g-g^{-1}, h-h^{-1}\right]=0$, for all $g, h \in G \backslash B$, since $B^{2}=1$. As $[G: B]=2$, we have $g=x a$ and $h=x b$, with $a, b \in B$ and $x \in G \backslash B$. Thus, since $a=a^{-1}$ and $b=b^{-1}$, we get

$$
\begin{aligned}
{\left[g-g^{-1}, h-h^{-1}\right] } & =\left[x a-a x^{-1}, x b-b x^{-1}\right] \\
& =x a x b-x a b x^{-1}-a b+a x^{-1} b x^{-1} \\
& -x b x a+x b a x^{-1}+b a-b x^{-1} a x^{-1} \\
& =x a x b+a x^{-1} b x^{-1}-x b x a-b x^{-1} a x^{-1} .
\end{aligned}
$$

Now, as $B$ is normal, we can write $a x=x a_{1}$ and $b x=x b_{1}$, for some $a_{1}, b_{1} \in B$. Thus, as $x^{2} \in B$ and $B$ is an elementary abelian 2-subgroup, we have $x^{4}=1$ and so $x^{2}=x^{-2}$. 
Hence,

$$
\left[g-g^{-1}, h-h^{-1}\right]=x^{2} a_{1} b+a b_{1} x^{-2}-x^{2} b_{1} a-b a_{1} x^{-2}=0,
$$

and we are done.

Now, we can give a complete characterization of the groups $G$ such that $R G^{-}$is commutative, when $\operatorname{char}(R) \neq 4$.

Theorem 3.3. Let $R$ be a commutative ring with unity with $\operatorname{char}(R) \neq 2,4$ and let $G$ be any group. Then $R G^{-}$is commutative if and only if one of the following conditions holds:

(i) $G$ is abelian;

(ii) $A=\langle g \in G \mid o(g) \neq 2\rangle$ is a normal abelian subgroup of $G$;

(iii) $G$ contains an elementary abelian 2-subgroup of index 2 .

Proof. Suppose that $G$ is non-abelian. If the elements of order not equal to 2 commute, then (ii) holds. Thus, we may assume that there exists one pair of elements of order not equal to 2 that do not commute and show that, in this case, (iii) holds.

First we consider the subgroup $B=\left\langle x y \mid x, y \in G, x^{2} \neq 1 \neq y^{2}\right\rangle$ generated by all the products of two elements of $G$ each of order not equal to 2 . We claim that $B$ is an elementary abelian 2-subgroup of $G$.

In fact, let $x, y \in G$ be such that $x^{2} \neq 1 \neq y^{2}$. Since, by Lemma $3.1,(x y)^{2}=1$, in order to establish the claim it is sufficient to show that any pair of generators of $B$ commute. Let $x_{i}, y_{i} \in G$ be such that $x_{i}^{2} \neq 1 \neq y_{i}^{2}, i=1,2$. Note that since Lemma 3.1 implies the equalities

$$
y_{1} x_{2}=x_{2}^{-1} y_{1}^{-1}, \quad x_{1} x_{2}^{-1}=x_{2} x_{1}^{-1}, \quad y_{1}^{-1} y_{2}=y_{2}^{-1} y_{1}, \quad x_{1}^{-1} y_{2}^{-1}=y_{2} x_{1},
$$

we have

$$
\left(x_{1} y_{1}\right)\left(x_{2} y_{2}\right)=x_{1} x_{2}^{-1} y_{1}^{-1} y_{2}=x_{2} x_{1}^{-1} y_{2}^{-1} y_{1}=\left(x_{2} y_{2}\right)\left(x_{1} y_{1}\right)
$$

and the claim is established.

Now, let $B_{1}$ be a maximal elementary abelian 2-subgroup of $G$ containing $B$. We shall show that $B_{1}$ has index 2 in $G$. Let $x B_{1}$ be a left coset of $B_{1}$ distinct from $B_{1}$. We claim that we may assume that $x^{2} \neq 1$. In fact, suppose that $x^{2}=1$. If $x b=b x$, for all $b \in B_{1}$, then $\left\langle B_{1}, x\right\rangle$ is an elementary abelian 2-subgroup of $G$ properly containing $B_{1}$. But this contradicts the maximality of $B_{1}$. Thus, there exists $b \in B_{1}$ such that $x b \neq b x$. As $x^{2}=b^{2}=1$, if $(x b)^{2}=1$, then we would get $x b=b x$ : a contradiction. Hence, $(x b)^{2} \neq 1$ and $x^{\prime}=x b$ is also a coset representative of $x B_{1}$.

Now let $x B_{1}$ and $y B_{1}$ be two cosets of $B_{1}$ and suppose, as we may by the above, that $x^{2} \neq 1 \neq y^{2}$. Then, by definition of $B$, we have that $x^{-1} y \in B \subset B_{1}$, and this says that $x B_{1}=y B_{1}$. Hence, $B_{1}$ is an elementary abelian 2-subgroup of index 2 in $G$ and (iii) holds.

The converse is a particular case of the previous lemma. 


\section{Skew symmetric elements when $\operatorname{char}(R)=4$}

Note that the proof of Theorem 3.3 depends on Lemmas 3.1 and 3.2, so it also holds when $\operatorname{char}(R)=4$, provided that $G$ does not contain subgroups isomorphic to $\mathcal{Q}_{8}$. Hence, from now on, we shall assume that $G$ contains at least one such subgroup. We shall see that the commutativity of the skew elements implies a quite restrictive condition on the group. To do so, we begin with some examples.

First, we note that a simple calculation shows that the group

$$
\langle g, h\rangle=\left\langle g, h \mid g^{4}=h^{4}=(g h)^{2}=\left(g h^{-1}\right)^{2}=1\right\rangle,
$$

of Lemma 2.2 (ii), contains the following elementary abelian 2-subgroup:

$$
A=\left\{1, g^{2}, h^{2}, g^{2} h^{2}, g h, g^{3} h, g h^{3}, g^{3} h^{3}\right\} .
$$

Thus, in this case, $\langle g, h\rangle$ is one of the groups described in Lemma 3.2.

We now proceed to show that the groups described in (iii) and (iv) of that lemma also give group algebras with commuting skew elements when $\operatorname{char}(R)=4$. To this end, we recall that it is sufficient to show that elements of the form $g-g^{-1}$, with $g \in G$, commute among themselves.

Example 4.1. Let $\mathcal{Q}_{8}=\left\langle a, b \mid a^{4}=1, b^{2}=a^{2}, b^{-1} a b=a^{-1}\right\rangle$ be the quaternion group of order 8 .

As $b^{-1} a b=a^{-1}$ and $b^{2}=a^{2}=a^{-2}$ we have

$$
b a^{-1}=a b=a^{-1} b^{-1}=b^{-1} a
$$

and

$$
a b^{-1}=b^{-1} a^{-1}=b a=a^{-1} b .
$$

Hence, if $\operatorname{char}(R)=4$, we obtain

$$
\begin{aligned}
{\left[a-a^{-1}, b-b^{-1}\right] } & =a b-a b^{-1}-a^{-1} b+a^{-1} b^{-1}-b a+b a^{-1}+b^{-1} a-b^{-1} a^{-1} \\
& =4(a b-b a) \\
& =0
\end{aligned}
$$

Since this holds for every pair of elements of $\mathcal{Q}_{8}$ with non-trivial squares, we find that $R \mathcal{Q}_{8}^{-}$is commutative.

Example 4.2. Let

$$
\begin{aligned}
G & =\left\langle a, b \mid a^{8}=1, b^{2}=a^{4}, b a=a^{-1} b^{-1}\right\rangle \\
& =\left\{1, a, a^{2}, a^{3}, a^{4}, a^{5}, a^{6}, a^{7}, b, a b, a^{2} b, a^{3} b, a^{4} b, a^{5} b, a^{6} b, a^{7} b\right\} .
\end{aligned}
$$

As the elements $a^{4}, a b, a^{3} b, a^{5} b$ and $a^{7} b$ have order 2 , in order to study the commutativity of $R G^{-}$it is sufficient to consider the elements $a-a^{7}=a-a^{-1}, a^{2}-a^{6}, a^{3}-a^{5}$, $b-a^{4} b=b-b^{-1}$ and $a^{2} b-a^{6} b=a^{2} b-a^{2} b^{-1}$. 
Clearly, the elements that depend only on $a$ commute among themselves. Also, as

$$
b^{-1} a=b^{3} a=b^{2} b a=b^{2} a^{-1} b^{-1}=a^{-1} b^{2} b^{-1}=a^{-1} b,
$$

we have

$$
\begin{aligned}
{\left[a-a^{-1}, b-b^{-1}\right] } & =a b-a b^{-1}-a^{-1} b+a^{-1} b^{-1}-b a+b a^{-1}+b^{-1} a-b^{-1} a^{-1} \\
& =a b-a b^{-1}-a^{-1} b+a^{-1} b^{-1}-a^{-1} b^{-1}+a b^{-1}+a^{-1} b-a b \\
& =0
\end{aligned}
$$

and

$$
\begin{aligned}
{\left[a-a^{-1}, a^{2} b-a^{2} b^{-1}\right] } & =a^{3} b-a^{3} b^{-1}-a b+a b^{-1}-a^{2} b a+a^{2} b a^{-1}+a^{2} b^{-1} a-a^{2} b^{-1} a^{-1} \\
& =a^{3} b-a^{3} b^{-1}-a b+a b^{-1}-a b^{-1}+a^{3} b^{-1}+a b-a^{3} b \\
& =0 .
\end{aligned}
$$

Similarly, $a^{3}-a^{5}$ commutes with $b-b^{-1}$ and with $a^{2} b-a^{2} b^{-1}$.

Finally, since

$$
\begin{aligned}
\left\langle a^{2}, b\right\rangle & =\left\{1, a^{2}, a^{4}, a^{6}, b, a^{2} b, a^{4} b, a^{6} b\right\} \\
& =\left\langle a^{2}, b \mid\left(a^{2}\right)^{4}=1, b^{2}=\left(a^{2}\right)^{2}, b^{-1} a^{2} b=b^{-2} a^{2}=a^{-2}\right\rangle \\
& \cong \mathcal{Q}_{8},
\end{aligned}
$$

by the example above it follows that the remaining pairs of elements commute provided that $\operatorname{char}(R)=4$.

Lemma 4.3. Let $G$ be a group with a subgroup isomorphic to $\mathcal{Q}_{8}$ and suppose that $R G^{-}$is commutative. Then $G$ is a 2 -group and its exponent is bounded by 8 .

Proof. Let $\mathcal{Q}_{8} \cong\langle x, y\rangle \subset G$ and suppose that there exists $g \in G$ with $g^{8} \neq 1$. Then, by Lemma 2.2, it follows immediately that $g$ commutes with all $h \in G$, such that $h^{2} \neq 1$. We claim that $(g x)^{2} \neq 1$. If not, then $1=(g x)^{2}=g^{2} x^{2}$, and we would get $g^{4}=1$, a contradiction. Similarly, $(g y)^{2} \neq 1$.

Now, by again applying Lemma 2.2 to $g x$ and $g y$, we either have that $g x$ and $g y$ commute or we obtain $(g x)^{8}=1$. In the case in which $g x$ and $g y$ commute, $g^{2} x y=$ $g x g y=g y g x=g^{2} y x$, and we obtain $x y=y x$ : a contradiction. On the other hand, if $(g x)^{8}=1$, then $1=g^{8} x^{8}=g^{8}$ : a contradiction. In conclusion, we have proved that $g^{8}=1$, for all $g \in G$.

Lemma 4.4. Let $G$ be a group with an abelian subgroup $A$ of exponent 8 and of index 2. Suppose that there exists $b \in G$ of order 4 , such that $b^{-1} a b=a^{3}$, for all $a \in A$, and such that $a^{o(a) / 2}=b^{2}$, for all $a \in A, a^{2} \neq 1$. Then

$$
G \cong\left\langle g, h \mid g^{8}=1, h^{2}=g^{4}, g h=h^{-1} g^{-1}\right\rangle \times E,
$$

where $E$ is an elementary abelian group. 
Proof. First, recall that every abelian group of finite exponent is a product of cyclic groups of prime power order (see, for example, $[4,(5.1 .2)$, p. 92]). Hence, as the exponent of $A$ is equal to 8 , we find that $A$ has a cyclic subgroup $\langle a\rangle$ of order 8 , as a direct factor.

We next show that $A$ is the direct product of $\langle a\rangle$ and an elementary abelian group. To this end, suppose that $A$ has another direct factor of order not equal to 2 . So, there exists an element $c$ of $A$ such that $c^{2} \neq 1$ and $\langle c\rangle \cap\langle a\rangle=\{1\}$. Then, as $c^{o(c) / 2}=b^{2}=a^{4}$, we have $1 \neq b^{2} \in\langle c\rangle \cap\langle a\rangle$ : a contradiction. Thus, $A=\langle a\rangle \times E$, with $E^{2}=1$.

Now we show that $G$ is the direct product of $\langle a, b\rangle$ and $E$. First note that $b \in G \backslash A$. In fact, if $b \in A$, then $b=b^{-1} b b=b^{3}$ and $b^{2}=1$, a contradiction since $o(b)=4$. Thus, as $A$ has index 2 in $G$, it follows that $G=A \cup A b$. So, as $A$ is abelian and for all $e \in E$, $b^{-1} e b=e^{3}=e$ holds, we find that $E$ is central in $G$ and, consequently, $E$ is normal in $G$. Hence, for all $g \in G$ we have that $g=a^{i} e^{j} b^{k}=a^{i} b^{k} e^{j}$ and $G=\langle a, b\rangle E$ follows. Moreover,

$$
g^{-1}\langle a, b\rangle g=b^{-k} e^{-j} a^{-i}\langle a, b\rangle a^{i} e^{j} b^{k}=b^{-k} a^{-i}\langle a, b\rangle a^{i} b^{k} \in\langle a, b\rangle,
$$

and $\langle a, b\rangle$ is normal in $G$. Finally, as $\langle a\rangle \cap E=\{1\}$ and $A b=G \backslash A$, it follows that $\langle a, b\rangle \cap E=\{1\}$.

It only remains to show that

$$
\langle a, b\rangle=\left\langle a, b \mid a^{8}=1, b^{2}=a^{4}, a b=b^{-1} a^{-1}\right\rangle .
$$

But, as $b^{-1} a b=a^{3}, a^{4}=b^{2}$ and $o(b)=4$, we have $a b=b a^{3}=b a^{4} a^{-1}=b^{3} a^{-1}=b^{-1} a^{-1}$, and the result follows.

With the results above at hand, a characterization of these groups can now be given.

Theorem 4.5. Let $G$ be a group of exponent 8, containing a copy of $\mathcal{Q}_{8}$ and let $R$ be a ring of characteristic 4. Then $R G^{-}$is commutative if and only if $G$ is isomorphic to the direct product of the group $\left\langle g, h \mid g^{8}=1, h^{2}=g^{4}, g h=h^{-1} g^{-1}\right\rangle$ and an elementary abelian group.

Proof. Note that in order to show that the condition is necessary it is sufficient to check that the hypothesis of Lemma 4.4 holds. Let $A=\langle g \in G \mid o(g)=8\rangle$. Then, by Lemma 2.2 , we have that $A$ is a normal abelian subgroup and, by hypothesis $A$ is non-trivial.

Clearly, $A \neq G$, as $G$ is not abelian. We next show that $A$ is of index 2 in $G$. To this end, we first show that if $a$ is an element of order 8 and $b \in G \backslash A$, then $b^{-1} a b=a^{-1}$ or $a^{3}$. Note that $a b \neq b a$. In fact, if $a b=b a$, then $(a b)^{4}=a^{4} b^{4}=a^{4} \neq 1$, and thus $a b \in A$ : a contradiction. Also note that, as $b^{4}=1$, it is sufficient to study the cases $o(b)=4$ and $o(b)=2$. If $o(b)=4$, then Lemma 2.2 (iii) holds for $a$ and $b$, and

$$
\langle a, b\rangle=\left\langle a, b \mid a^{8}=1, b^{2}=a^{4}, a b=b^{-1} a^{-1}\right\rangle .
$$

Thus, $b^{-1} a b=b^{-2} a^{-1}=a^{-5}=a^{3}$.

Now, suppose that $o(b)=2$. If $(a b)^{2}=1$, then $a b=b a^{-1}$, and $b^{-1} a b=a^{-1}$. On the other hand, if $(a b)^{2} \neq 1$, then Lemma 2.2 (iii) holds for $a$ and $a b$, since $a b \neq b a$. Hence,

$$
b^{-1} a b=b a b=a^{-1}(a b)^{2}=a^{-1} a^{4}=a^{3},
$$

and $b^{-1} a b=a^{-1}$ or $a^{3}$. 
Finally, let $g, h \in G \backslash A$ and $a \in A$, such that $o(a)=8$. Suppose that $g h \in G \backslash A$. Then, $\left(g h^{-1}\right)^{-1} a\left(g h^{-1}\right)$ is equal to either $a^{-1}$ or $a^{3}$. On the other hand, we also have that $\left(g h^{-1}\right)^{-1} a\left(g h^{-1}\right)=h\left(g^{-1} a g\right) h^{-1}$ is equal to either $h a^{-1} h^{-1}$ or $h a^{3} h^{-1}$, and thus $\left(g h^{-1}\right)^{-1} a\left(g h^{-1}\right)$ is equal to either $a$ or $a^{-3}$. So, $a^{2}=1$ : a contradiction. Hence, $g h^{-1} \in A$, and $g A=h A$. It follows that the index of $A$ in $G$ is equal to 2 .

Now, let $b$ be an element of $G \backslash A$ of order 4 . This element exists since $G$ contains a copy of $\mathcal{Q}_{8}$. We have already shown that if $a \in A$ is an element of order 8 , then $b^{-1} a b=a^{3}$. Thus, as $A$ is abelian and $A$ is generated by the elements of order 8 , we have $b^{-1} a b=a^{3}$, for all $a \in A$. Also, we have shown that if $a$ is an element of order 8 , then $b^{2}=a^{4}=a^{o(a) / 2}$. It remains to prove that $b^{2}=a^{2}$, for all $a \in A$ with $o(a)=4$. In fact, by Lemma 2.1, $a b=b a, a b=b^{-1} a^{-1}$, or $\langle a, b\rangle \cong \mathcal{Q}_{8}$ follows. If $\langle a, b\rangle \cong \mathcal{Q}_{8}$, we are clearly done. Hence suppose that either the first or the second possibility occurs. As $b^{-1} a b=a^{3}=a^{-1}$, we have that $a b=b a^{-1}$, and thus either $b a=a b=b a^{-1}$ or $b^{-1} a^{-1}=a b=b a^{-1}$. Hence, $a^{2}=1$ or $b^{2}=1$ : a contradiction. The necessity now follows from Lemma 4.4.

We next prove the sufficiency. Suppose that

$$
H=\left\langle g, h \mid g^{8}=1, h^{2}=g^{4}, g h=h^{-1} g^{-1}\right\rangle
$$

and $G=H \times E$, where $E^{2}=1$. We first show that the commutativity of $R H^{-}$implies the commutativity of $R G^{-}$. In fact, $R G^{-}$is commutative if the elements of the form $g-g^{-1}$, where $g \in G, g^{2} \neq 1$, commute among themselves. But $g-g^{-1}=h e-h^{-1} e^{-1}$, where $h \in H, h^{2} \neq 1$, and $e \in E$. Hence, as $E^{2}=1$, it follows that $g-g^{-1}=\left(h-h^{-1}\right) e$. So, as $E$ is central in $G$, we find that if the terms of the form $h-h^{-1}$, with $h \in H, h^{2} \neq 1$, commute among themselves, then the terms of the form $g-g^{-1}$, with $g \in G, g^{2} \neq 1$, also commute among themselves. Hence, the commutativity of $R G^{-}$follows from the commutativity of $R H^{-}$.

To complete the proof it is sufficient to note that the commutativity of $\mathrm{RH}^{-}$has been proved in Example 4.2.

Theorem 4.6. Let $G$ be a group of exponent 4 and let $R$ be a ring of characteristic 4 . Then $R G^{-}$is commutative if and only if, for every pair of elements $g$ and $h$ in $G$ such that $g^{2} \neq 1 \neq h^{2}$, one of the following holds:

(i) $\langle g, h\rangle$ is abelian;

(ii) $\langle g, h\rangle=\left\langle g, h \mid g^{4}=h^{4}=(g h)^{2}=\left(g h^{-1}\right)^{2}=1\right\rangle$;

(iii) $\langle g, h\rangle=\left\langle g, h \mid g^{4}=1, h^{2}=g^{2}, g h=h g^{-1}\right\rangle \cong \mathcal{Q}_{8}$.

Proof. The necessity of the condition follows immediately from Lemma 2.2. To prove sufficiency, as $R G^{-}$is generated, as an $R$-module, by the elements of the form $g-g^{-1}$, it is sufficient to prove that $\left[g-g^{-1}, h-h^{-1}\right]=0$, for all $g, h \in G$ such that $g^{2} \neq 1 \neq h^{2}$.

Let $g$ and $h$ be such elements of $G$ and suppose that (i), (ii) or (iii) holds. If case (i) holds, the result is immediate. 
Suppose that (ii) holds. Then

$$
\begin{aligned}
{\left[g-g^{-1}, h-h^{-1}\right] } & =g h-g h^{-1}-g^{-1} h+g^{-1} h^{-1}-h g+h g^{-1}+h^{-1} g-h^{-1} g^{-1} \\
& =h^{-1} g^{-1}-h g^{-1}-h^{-1} g+h g-h g+h g^{-1}+h^{-1} g-h^{-1} g^{-1} \\
& =0 .
\end{aligned}
$$

Now, if (iii) holds, then commutativity follows from Example 4.1.

In order to have a better understanding of the groups obtained above, we shall describe all their subgroups generated by two elements.

Proposition 4.7. Let $G$ be a group of exponent 4 such that $R G^{-}$is commutative. Let $g$ and $h$ be elements of $G$, such that $g^{2} \neq 1=h^{2}$. Then, one of the following holds:

(i) $\langle g, h\rangle$ is abelian;

(ii) $\langle g, h\rangle$ is isomorphic to the group described in Lemma 2.2 (ii);

(iii) $\langle g, h\rangle \cong\left\langle a, b \mid a^{4}=b^{2}=1, b a b=a^{-1}\right\rangle$, the dihedral group of order 8 .

Proof. We suppose that $g h \neq h g$ and we prove that either (ii) or (iii) holds. If $(g h)^{2} \neq 1$, then we can apply Lemma 2.2 to $g$ and $g h$, and one of the following three possibilities occurs: $g g h=g h g$, where $g h=h g$, or $\langle g, g h\rangle=\langle g, h\rangle$ is isomorphic to $\left\langle g, h \mid g^{4}=h^{4}=(g h)^{2}=\left(g h^{-1}\right)^{2}=1\right\rangle$, or $\langle g, h\rangle$ is isomorphic to $\mathcal{Q}_{8}$. But the first and the last possibility cannot occur, since $g h \neq h g$ and $\mathcal{Q}_{8}$ cannot be generated by an element of order 4 and an element of order 2. Thus, (ii) holds.

Now, if $(g h)^{2}=1$, then $g h=h^{-1} g^{-1}=h g^{-1}$, and

$$
\begin{aligned}
\langle g, h\rangle & =\left\langle g, h \mid g^{4}=h^{2}=1, g h=h g^{-1}\right\rangle \\
& \cong D_{4} .
\end{aligned}
$$

So, (iii) holds and the result follows.

Proposition 4.8. Let $G$ be a group of exponent 4 such that $R G^{-}$is commutative. Let $g$ and $h$ be elements of $G$ of order 2. Then $\langle g, h\rangle$ is either abelian or isomorphic to the dihedral group of order 8 .

Proof. Assume that $g h \neq h g$. Then $(g h)^{2} \neq 1$. In fact, if $(g h)^{2}=1$, then $g h=h^{-1} g^{-1}=h g$. Now, applying Lemma 4.7 to the elements $g h$ and $g$, we have the following three possibilities for the group $\langle g h, g\rangle=\langle g, h\rangle:\langle g, h\rangle$ is abelian or is isomorphic to the group (ii) of Lemma 2.2 or is isomorphic to $D_{4}$. But, by hypothesis, $\langle g, h\rangle$ is non-abelian. Finally, as in the proof of Lemma 4.7 , it follows that $\langle g, h\rangle \cong D_{4}$, since $(g h g)^{2}=g h g g h g=1$.

As an illustration, we list the groups $G$ of order $2^{n}$, containing a subgroup isomorphic to $\mathcal{Q}_{8}$, with $n \leqslant 5$, such that $R G^{-}$is commutative, for a ring $R$ of characteristic 4 . We use the notation of [5] to identify the groups given below. 
(i) $n=3 \mathcal{Q}_{8}=8 / 5$.

(ii) $n=4$
(a) $16 / 7=\mathcal{Q}_{8} \times C_{2}$, where $C_{2}$ denotes the cyclic group of order 2 .
(b) $16 / 8=\left\langle a, b, c \mid a^{2}=b^{2}=c^{2}=1, a b c=b c a=c a b\right\rangle$.

(ii) $n=5$
(a) $32 / 9=(16 / 7) \times C_{2}$.
(b) $32 / 10=(16 / 8) \times C_{2}$.
(c) $32 / 39=\left\langle a, b, c \mid a^{4}=b^{4}=c^{2}=1, a b=b a, a c=c a^{-1}, b c=c a^{2} b^{-1}\right\rangle$.
(d) $32 / 42=\langle a, b, c, d| a^{4}=1, a^{2}=b^{2}=c^{2}=d^{2}, a b=b a^{-1}, c d=d c^{-1},(a, c)=$ $(a, d)=(b, c)=(b, d)=1\rangle$.
(e) $32 / 43=\langle a, b, c, d| a^{2}=b^{4}=c^{2}=1, b^{2}=d^{2}, a c=c b^{2} a, b c=c b^{-1}, a d=$ $\left.d b^{2} a,(a, b)=(b, d)=(c, d)=1\right\rangle$.

Acknowledgements. The authors are grateful to Professor Antonio Giambruno for helpful conversations while this work was done. This work was partly supported by FAPESP, Procs. 99/04759-2, 03/07246-3 and 2000/07291-0 and CNPq 300243/79-0(RN).

\section{References}

1. O. Broche Cristo, Commutativity of symmetric elements in group rings, J. Group Theory, in press.

2. A. Giambruno and C. Polcino Milies, Unitary units and skew elements in groups algebras, Manuscr. Math. 111 (2003), 195-209.

3. A. Giambruno and S. K. Sehgal, Lie nilpotence of group rings, Commun. Alg. 21 (1993), 4253-4261.

4. W. R. Sсотt, Group theory (Prentice-Hall, Englewood Cliffs, NJ, 1964).

5. A. D. Thomas and G. V. Woods, Group tables (Shiva, Exeter, 1980). 